Annuaire suisse de politique de développement

$21 \mid 2002$

Agriculture suisse et mondialisation

\title{
Le pouvoir des ONG sur les entreprises : pression, partenariat, évaluation
}

Antoine Mach

\section{(2) OpenEdition}

1 Journals

Édition électronique

URL : http://journals.openedition.org/aspd/936

DOI : 10.4000/aspd.936

ISSN : 1663-9669

Éditeur

Institut de hautes études internationales et du développement

\section{Édition imprimée}

Date de publication : 1 avril 2002

Pagination : 109-129

ISSN : 1660-5934

\section{Référence électronique}

Antoine Mach, «Le pouvoir des ONG sur les entreprises : pression, partenariat, évaluation », Annuaire suisse de politique de développement [En ligne], 21 | 2002, mis en ligne le 06 septembre 2012, consulté le 08 septembre 2020. URL : http://journals.openedition.org/aspd/936 ; DOI : https://doi.org/10.4000/ aspd.936

Ce document a été généré automatiquement le 8 septembre 2020

(c) The Graduate Institute I Geneva 


\title{
Le pouvoir des ONG sur les entreprises : pression, partenariat, évaluation
}

\author{
Antoine Mach
}

\section{Introduction}

1 Face aux inquiétudes liées à la globalisation de l'économie et aux implications du modèle de la valeur actionnariale, les entreprises multinationales prennent des initiatives de responsabilité sociale. Il s'agit de discours et d'actions qui ont pour but de montrer au public que si leur mission première est d'apporter des bénéfices à leurs actionnaires au prix de délocalisations et de licenciements, ces entreprises contribuent néanmoins au bien-être des populations dans un rayonnement dépassant leurs clients et collaborateurs. Les organisations non gouvernementales (ONG) jouent un rôle important dans ce processus. En amont, elles sensibilisent le public par des manifestations et des campagnes de pression visant les gouvernements et, de plus en plus directement, les entreprises. En aval, les ONG collaborent avec certaines entreprises dans le cadre de partenariats visant par exemple l'amélioration des conditions de travail dans les pays producteurs ou la lutte contre la corruption.

2 Les campagnes ciblées et les partenariats peuvent être considérés comme des relations directes entre ONG et entreprises, celles-ci entretenant également des relations indirectes autour des processus de décision inscrits dans le champ politique institutionnel. Cet article se concentre sur la première catégorie. Ce type de relations a été décrit comme relevant d'une régulation civile de la société, distincte de la régulation légale (Bendell 2000), d'une "autoréglementation des entreprises » (Utting 2001), de «la manifestation d'un droit mondial sans Etat» et «de régimes de gouvernance privée » (Teubner 1997). Un débat a cours actuellement sur l'efficacité politique de la régulation civile, sur les réels changements sociaux à attendre des campagnes de pression et des partenariats qui relient ONG et entreprises, ainsi que des 
politiques publiques incitatives que ce type de mécanisme peut inspirer. Certains pensent qu'il faut désormais concentrer les efforts de recherche et de sensibilisation du public sur ce modèle, investir les marchés, passer par les consommateurs et les actionnaires pour influencer directement le comportement de l'entreprise et l'amener à servir l'intérêt public au-delà des exigences légales. D'autres voient au contraire dans la responsabilité sociale de l'entreprise un leurre, un mythe, jugeant par trop improbable la coïncidence des intérêts d'une entreprise privée et des intérêts de la collectivité. Ils en appellent plutôt à un renforcement des prérogatives du secteur public et de la pression dans les institutions nationales et internationales en faveur d'une plus grande contrainte légale sur les acteurs économiques.

Cet article porte sur les relations directes entretenues par des ONG et des entreprises à propos d'une douzaine d'enjeux socio-économiques de dimension globale depuis 1970. Il propose également une typologie des relations entre ONG et entreprises et finit sur une évaluation des opportunités politiques liées aux différents types de relations proposés ${ }^{1}$. La majorité des cas étudiés concernent des relations entre des entreprises et des ONG suisses à propos d'enjeux touchant les pays en développement. Ces enjeux ont été retenus en fonction de l'information disponible et dans un souci de variété et de représentativité (différentes industries concernées), mais non d'exhaustivité. Les résultats sont basés sur des informations publiques d'une part (articles, site Internet) et sur des interviews d'autre part (10 entreprises, $20 \mathrm{ONG}$ ).

Par «ONG », on entend des organisations à but non lucratif actives dans la défense des droits de l'homme, la coopération au développement, l'action humanitaire et la protection de l'environnement. Par « entreprises multinationales ", on entend bien sûr les grands groupes privés actifs dans des dizaines de pays et cotés sur différentes places financières, mais aussi des entreprises de taille moyenne et d'ancrage national entretenant cependant des relations avec des partenaires situés à l'étranger.

\section{Résultats}

5 Les relations entre ONG et entreprises ont souvent été décrites à l'aide de l'alternative confrontation/partenariat (cf. Bendell 2000 ; Elkington \& Fennell 1998 ; Joerchel 1997). Dans cet article, on part de l'opposition pression/partenariat («pression » nous parait préférable à "confrontation", car ce terme indique mieux le caractère parfois unilatéral de ces relations.) Il est possible de distinguer différents types de pressions et de partenariats reliant ONG et entreprises.

- Pression institutionnelle: des ONG effectuent un travail de lobbying au sein des institutions politiques nationales et internationales en faveur de l'adoption de législations contraignantes à l'égard des entreprises. Ainsi, des organisations militent en faveur de l'adoption d'une clause sociale dans le cadre de l'Organisation mondiale du commerce, qui devrait permettre une amélioration des conditions de travail à l'échelle internationale. Au sein de la Sous-commission des Nations Unies pour les droits de l'homme, une composante du Groupe de travail sur les sociétés transnationales propose l'adoption d'une norme contraignante à l'égard des entreprises en matière de droits de l'homme ${ }^{2}$. Enfin, lors de la récente révision des Principes directeurs de l'OCDE à l'attention des entreprises multinationales ${ }^{3}$, des $\mathrm{ONG}$ ont proposé de rendre ces principes légalement contraignants ${ }^{4}$. - Pression informelle : des ONG participent à des manifestations qui, de Seattle à Gênes en passant par Davos, expriment une inquiétude et une insatisfaction devant la mondialisation 
de l'économie et le pouvoir des multinationales. Les critiques et demandes véhiculées à ces occasions visent les gouvernements des pays les plus industrialisés, les organisations internationales comme l'OMC, le FMI ou la Banque mondiale ainsi que les entreprises multinationales. Elles s'inscrivent dans le mouvement dit « antimondialisation ».

- Pression ciblée : des ONG lancent des campagnes visant certaines entreprises et certains aspects de leur comportement. Ces ONG cherchent à promouvoir des changements ponctuels dans la conduite des entreprises en s'appuyant sur les relais médiatiques, la sensibilisation du public et la participation des consommateurs.

- Partenariat orienté vers l'activité de l'entreprise : des ONG collaborent avec des entreprises pour la définition et la mise en œuvre de programmes sociaux impliquant une participation volontaire du secteur privé : labels de commerce équitable, code de conduite, certification sociale. Les ONG apportent leur soutien et leur expertise à des initiatives menées par des entreprises.

- Partenariat orienté vers l'activité de l'ONG : d'autres partenariats ne portent pas sur un changement dans le circuit économique auquel participe l'entreprise mais sur l'activité de l'ONG : ce sont des programmes de sponsoring ou de marketing lié à une cause (cf. 1 franc par produit vendu attribué à des projets sociaux).

6 Cet article se concentre sur les pressions ciblées et sur les partenariats orientés vers l'activité de l'entreprise, pour deux raisons : ces types de relations paraissent moins connus et étudiés que la pression institutionnelle et la pression informelle; cette zone nous semble présenter des opportunités politiques particulièrement intéressantes pour les ONG et pour les différents acteurs des droits de l'homme et du développement.

\section{Etude de cas : pressions ciblées et partenariats orientés vers l'activité de l'entreprise}

\section{Commerce de lait pour bébé}

Depuis 1974, des $\mathrm{ONG}^{5}$ adressent des reproches à l'industrie alimentaire au sujet de la commercialisation de lait artificiel dans les pays du tiers-monde. Selon les sources, ces reproches visent le principe même de la commercialisation de lait artificiel dans ces pays, ou seulement les méthodes de vente de ce produit. Certains considèrent qu'il est illégitime de vendre du lait artificiel dans des pays pauvres, à la fois pour des raisons de santé (le lait mal dosé ou mélangé à de l'eau insalubre menace la santé des bébés) et pour des raisons économiques (le lait maternel est gratuit, le lait en poudre coûte cher). Un grand nombre d'entreprises sont visées et citées dans des rapports, ainsi, pour la Suisse, Migros ${ }^{6}$. Leader mondial sur le marché du lait en poudre, Nestlé constitue cependant la cible principale des ONG actives sur ce dossier. En 1981, l'Organisation mondiale de la santé (OMS) adopte un Code international de commercialisation des substituts du lait maternel, auquel Nestlé et d'autres producteurs adhéreront dans les années qui suivent, s'imposant ainsi une série de limites dans leurs pratiques publicitaires. Par la suite, les reproches visant l'industrie alimentaire ont diminué, mais n'ont pas totalement disparu, des controverses se manifestant ici et là quant à l'application de ce code et à son respect par l'industrie. Depuis 1999, Nestlé publie régulièrement un rapport sur les actions entreprises en relation avec le code de l'OMS, actions «qui peuvent impliquer des partenariats avec les ONG qui soutiennent des 
projets destinés à fournir de l'eau non polluée, une nourriture convenable, de l'hygiène et une infrastructure médicale. $»^{7}$

\section{Conditions de production de la banane}

8 Au milieu des années 1970, à Frauenfeld, un groupe de femmes organise des actions de sensibilisation du public aux conditions de travail en vigueur dans les grandes plantations de bananiers contrôlées par les multinationales. Ces femmes achètent des bananes à la Migros et les revendent 20 centimes plus cher sur le trottoir devant le magasin. Ainsi, elles veulent montrer qu'en payant un prix légèrement supérieur, les consommateurs pourraient acquérir des fruits produits dans de meilleures conditions humaines et sociales. Par cette démarche essentiellement symbolique, les Bananenfrauen $^{8}$ contribuent à la diffusion de l'idée du commerce équitable. En 1981, la Déclaration de Berne lance une campagne qui porte à la fois sur la faim et sur les conditions de travail dans les pays du tiers-monde ; les conditions de travail en vigueur dans les plantations d'ananas contrôlées par des multinationales occidentales aux Philippines sont dénoncées. Cette campagne vise aussi les entreprises qui distribuent les ananas en Suisse, comme Migros. Dans un premier temps, celle-ci dénonce le bienfondé de la campagne, pour ensuite s'engager dans un dialogue avec ses animateurs. En 1983, certaines des plus grandes ONG suisses ${ }^{9}$ négocient avec Migros, qui est elle-même intervenue auprès de Del Monte, une clause sociale régissant la production d'ananas aux Philippines. La Clause sociale Migros-Del Monte représente l'une des premières expériences de contrôle indépendant de l'application d'une norme sociale privée dans le contexte du commerce international ${ }^{10}$.

\section{Lutte contre le blanchiment d'argent}

Les auteurs de détournements de fonds publics sollicitent des établissements bancaires dans le monde entier, et notamment en Suisse, pour leur confier leur «magot». Constatant que cette évasion de capitaux s'opère au détriment des dépenses publiques et des intérêts des populations des pays d'origine, des ONG demandent aux banques de veiller strictement à refuser une telle clientèle. L'association Action place financière suisse (Aktion Finanzplatz Schweiz), créée en 1978, exerce une pression sur les établissements jugés complaisants en la matière. Avec la Déclaration de Berne, elle a ainsi publié en 1981 un Manifeste pour une Suisse sans capitaux en fuite ${ }^{11}$. D'autres actions de sensibilisation du public à la problématique du blanchiment d'argent ont été menées en Suisse au cours des vingt dernières années. En novembre 2000, une douzaine de banques actives à l'échelle internationale, dont Crédit Suisse Group et UBS, ont adopté les Principes de Wolfsberg, en partenariat avec l'ONG Transparency International, dans le but de participer plus efficacement à la lutte contre le blanchiment d'argent.

\section{Accès aux médicaments essentiels}

Des ONG demandent à l'industrie pharmaceutique de jouer un plus grand rôle dans la santé publique des pays en développement en améliorant l'accès aux médicaments permettant de traiter les maladies infectieuses. Elles prient les entreprises d'orienter une partie de leurs investissements en recherche et développement vers des maladies 
tropicales comme la malaria ou la maladie du sommeil. Les ONG militent également pour une baisse du prix de vente des médicaments actifs contre le sida. Ces questions ont été abordées, notamment, par le réseau Health Action International dès 1981, par la Déclaration de Berne avec Medicus Mundi dans le bulletin Med in Switzerland de 1987 à 1997, et par Médecins sans frontières (MSF) dont la Campagne pour l'accès aux médicaments essentiels a démarré en 1999. Parallèlement, certaines entreprises pharmaceutiques ont établi des partenariats avec des ONG : les Principes directeurs applicables aux dons de médicaments ont été adoptés par les agences internationales actives dans la santé publique, des ONG comme MSF, Oxfam, Caritas, le CICR (en 1996), et par les plus grandes entreprises pharmaceutiques (en 1999) ${ }^{12}$; la Fondation Novartis pour un développement durable a collaboré avec des organisations comme Helvetas ou Terre des Hommes - Suisse sur des projets de développement ${ }^{13}$; le Global Forum for Health Research ${ }^{14}$ réunit des experts gouvernementaux et non gouvernementaux ainsi que des représentants du secteur privé autour de questions liées à la recherche en matière de santé à l'échelle globale.

\section{Relations avec le régime d'apartheid}

11 Dans les années 1980, des $\mathrm{ONG}^{15}$ demandent aux industries et aux banques suisses de suspendre leurs activités commerciales et financières en relation avec l'Afrique du Sud, alors sous régime d'apartheid. Tout comme de nombreux gouvernements et les Nations Unies, ces ONG estiment que le boycott est la voie à suivre pour soutenir les efforts visant l'instauration de la démocratie en Afrique du Sud. Après la chute du régime d'apartheid, ces ONG estiment que le boycott a contribué à ce changement. Les entreprises concernées, elles, se rangent du côté de la politique définie par la Confédération suisse en la matière ${ }^{16}$ : elles considèrent que le boycott n'est pas une solution efficace pour faire chuter cette dictature, que la suspension des relations économiques est surtout préjudiciable aux populations les plus pauvres alors que leur maintien permet des contacts et un développement économique propices à l'avancement démocratique. Malgré des tentatives de dialogue menées à l'époque par des ONG proches des Eglises, il n'y a pas eu d'accord entre ONG et entreprises sur ce dossier ${ }^{17}$.

\section{Extraction du pétrole et droits de l'homme}

Les compagnies pétrolières font régulièrement face à des critiques et sollicitations d'ONG à propos de la situation des droits de l'homme dans les pays producteurs. Ainsi, des ONG ont demandé à Shell de suspendre ses activités au Nigeria au milieu des années 1990, alors qu'Amnesty International demandait en vain à l'entreprise d'intervenir auprès du gouvernement nigérian afin qu'il renonce à l'exécution de neuf activistes d'une organisation militant pour la protection de l'environnement et l'autodétermination du peuple des Ogoni, le Movement for the Survival of Ogoni People $(1995)^{18}$. De son côté, British Petroleum a dû faire face à des critiques portant sur les liens existant entre l'agence de sécurité privée mandatée pour protéger ses installations et les forces armées colombiennes. Troisième exemple : Unocal et Total ont reçu des demandes de suspension de leurs activités en Birmanie, celles-ci impliquant des relations jugées trop étroites avec le régime militaire en place (cf. Avery 2000). Les entreprises concernées ont réagi de différentes manières à ces 
requêtes. Certaines, comme Shell et BP, abordent ouvertement le rôle du secteur privé en matière de droits de l'homme dans leur communication d'entreprise. Ainsi Shell cite un rapport d'Amnesty International : «Amnesty International in its 6 November 1996 report (Nigeria: Time to end the contempt for human rights) states that : "In its approaches in recent years to Shell and other trans-national companies with significant investments in Nigeria, Amnesty International has appealed to them to acknowledge their responsibility to do ail that they can to uphold human rights under the Universal Declaration of Human Rights. Only Shell has done so to date." ${ }^{19}$ Cependant, on n'a pas constaté l'établissement de réels partenariats entre ONG et compagnies pétrolières en matière de droits de l'homme.

\section{Conditions de production du café}

Depuis les années 1970, en Suisse et en Europe, les Magasins du monde proposent au consommateur des produits issus d'un commerce équitable (achats directs auprès de coopératives, revenus supérieurs à la moyenne, contrats de longue durée). En 1987, la Communauté de travail des œuvres d'entraide lance un Appel en faveur du commerce équitable qui est soutenu par 100'000 citoyens; précisons que cet appel ne s'accompagne pas d'une pression visant directement des entreprises. En 1992, suivant les pionniers hollandais, les principales ONG suisses créent la Fondation Max Havelaar, qui a pour vocation de permettre la diffusion de produits du commerce équitable dans les réseaux de la grande distribution ${ }^{20}$. Très vite, Migros et Coop se montrent intéressées à l'idée de commercialiser les produits portant ce label. Les produits Max Havelaar (café, chocolat, sucre, miel, bananes, thé, jus d'orange, riz) sont vendus dans les grandes surfaces suisses et européennes avec un succès croissant. La Suisse est le pays européen où ce succès est le plus important en termes de parts de marché du café labellisé Max Havelaar, avec 3\%, devant les Pays-Bas $(2,7 \%)$, le Danemark $(1,8 \%)$ et l'Angleterre $(1,5 \%)^{21}$.

\section{Conditions de production des tapis d'Orient}

Dès la fin des années 1980, médias et ONG internationaux diffusent des informations relatives aux conditions de travail dans la fabrication des tapis en Asie. Le problème du travail des enfants suscite une grande émotion dans le public. En 1995, Action de Carême, la Déclaration de Berne, Pain pour le prochain et Swissaid s'associent à une organisation suisse de distributeurs de tapis, IGOT (Association pour un commerce loyal $\mathrm{du}$ tapis d'Orient), pour créer le label STEP ${ }^{22}$. Les commerces arborant ce label s'engagent à promouvoir des conditions de travail équitables dans les pays producteurs comme l'Inde, le Népal, le Pakistan, le Maroc ou l'Iran, ce qui implique la réduction du travail des enfants et surtout l'amélioration du sort des travailleurs adultes. La concrétisation des engagements couverts par le label STEP fait l'objet d'un contrôle indépendant impliquant des ONG locales avec la Fondation STEP en Suisse. Dans les pays germaniques et anglo-saxons, une initiative similaire est menée sous le label Rugmark. 


\section{Impact social des grands barrages} Greenpeace et le WWF lancent la Campagne contre une participation de la Suisse à la construction du barrage des Trois Gorges en Chine ${ }^{23}$. Des actions similaires sont menées pour les barrages d'Illisu en Turquie et de Mahesvar en Inde. Selon les ONG, ces projets entraînent de tels effets négatifs sur le plan social et environnemental qu'il convient de s'y opposer. Leurs campagnes visent d'abord les autorités gouvernementales suisses, et en particulier la Garantie contre les risques à l'exportation ${ }^{24}$. Mais elles visent aussi directement certaines entreprises suisses impliquées dans les projets, comme ABB, Sulzer ou le Crédit Suisse Group. La pression ciblée des ONG sur les entreprises se traduit par des communiqués de presse, des lettres ouvertes, un appel à l'envoi massif de cartes postales à l'entreprise ainsi que des interventions à l'assemblée générale des actionnaires, parfois avec l'appui d'investisseurs institutionnels comme la CIA, caisse de pension des fonctionnaires genevois, qui en 1998 a soutenu une démarche menée au sein de l'assemblée générale d'ABB à propos du barrage des Trois Gorges. Les ONG actives sur ce dossier et $\mathrm{ABB}$ ont eu l'occasion de dialoguer à quelques occasions, sans pour autant trouver un terrain d'entente au niveau suisse. A l'échelle internationale, en revanche, une collaboration entre secteur privé, ONG et autorités gouvernementales s'est établie en 1998 dans le cadre de la Commission mondiale sur les barrages, un organe créé sur l'initiative de la Banque mondiale, où $A B B$ et la Déclaration de Berne sont représentés ${ }^{25}$. Cette commission émet des recommandations quant à l'impact social et environnemental des grands ouvrages hydroélectriques, en particulier sur la question du déplacement des populations concernées et sur celle de l'octroi de compensations correspondantes.

\section{Tourisme en Birmanie}

De nombreux observateurs et institutions estiment que la Birmanie, ou Myanmar, est soumise à un régime auteur de violations massives et répétées des droits de l'homme. La Birmanie est également une destination de vacances appréciée des touristes occidentaux. Se pose donc la question de l'impact du tourisme sur la situation des droits de l'homme dans ce pays. Pour certaines ONG, comme l'Association SuisseBirmanie $^{26}$ et le Groupe de travail tourisme et développement (AKTE) ${ }^{27}$, il convient de boycotter cette destination car le tourisme a pour effet de renforcer le régime en place à travers les capitaux qu'il charrie. En Suisse, AKTE a mené campagne en 1998 contre les agences de voyages dépendant de Migros et Coop parce que celles-ci proposaient à leurs clients des vacances organisées en Birmanie. Cette pression semble avoir atteint son objectif, dans la mesure où Migros et Coop ont retiré ces offres du marché. Dans un échange de correspondance avec AKTE, Migros précise cependant qu'elle met en question l'efficacité des boycotts sur l'avancement des droits de l'homme et de la démocratie. Selon Migros, « un contact maintenu avec la population birmane à travers le tourisme contribue autant à faire évoluer les choses positivement $»^{28}$. Sur ce point, Migros est rejointe par Amnesty International - Suisse et son groupe Economie et droits humains. S'il n'hésite pas à dénoncer les violations massives des droits humains, ce groupe s'efforce aussi d'instruire les tour-opérateurs et de les sensibiliser aux enjeux politiques locaux «afin que les voyageurs puissent choisir leurs destinations en connaissance de cause $»^{29}$. Dans cet esprit, Amnesty International - qui en principe ne 
se prononce pas sur la pertinence des boycotts - approche depuis 2000 certaines agences de voyages pour les inciter à informer leurs clients de façon exhaustive : la situation des droits humains dans les pays de villégiature doit être clairement présentée à la clientèle. Initiative comparable, le label Tourism for Development a été lancé en 1998 depuis la France pour permettre aux voyageurs de favoriser les hôteliers qui s'impliquent dans le développement économique et social de leur pays en versant $1 \%$ des revenus des nuitées à des projets menés par des $\mathrm{ONG}^{30}$; ce label est soutenu par des compagnies d'aviation et par des agences de voyages.

\section{Conditions de production de vêtements}

17 En 1999, la Déclaration de Berne, Pain pour le prochain et Action de Carême lancent la Campagne Clean Clothes - Pour des habits produits dans la dignité, une initiative d'origine hollandaise qui fait suite à une campagne similaire ayant visé les marques et distributeurs de chaussures de sport (Let's go fair). La Campagne Clean Clothes demande aux marques et distributeurs de vêtements d'intervenir auprès de leurs fournisseurs et sous-traitants afin que ceux-ci appliquent les normes fondamentales de l'Organisation internationale du travail, y compris celles relatives aux droits syndicaux. Un code de conduite Clean Clothes est rédigé à cet effet. La campagne invite les consommateurs à envoyer des cartes postales ad hoc aux entreprises suisses concernées. Plus de 60'000 cartes atteignent leurs destinataires. Ceux-ci réagissent diversement, certains faisant la sourde oreille, d'autres annonçant des initiatives qui répondent plus ou moins aux attentes des ONG. Alors que la campagne de pression se poursuit aujourd'hui, trois des entreprises visées ont établi un partenariat avec les ONG organisatrices de la Campagne Clean Clothes: Migros, Veillon et Switcher. Ce partenariat lancé en 2000 consiste en un projet pilote de contrôle indépendant de l'application du code de conduite Clean Clothes dans les pays producteurs ${ }^{31}$.

\section{Débat sur la peine de mort}

Le 7 janvier 2000, Benetton lance une campagne de publicité qui a pour cadre les EtatsUnis. Intitulée "We, on death row», elle présente des portraits photographiques de condamnés à mort ainsi que des éléments biographiques. Selon l'entreprise italienne, la campagne a pour objectif de promouvoir le débat sur la peine capitale, de rendre une apparence humaine aux prisonniers des couloirs de la mort, de "rappeler aux gens respectables qui sont toujours sûrs d'avoir raison que le débat concerne des hommes et des femmes de chair et de sang et non des personnages virtuels que l'on peut éliminer d'un simple clic comme dans un jeu vidéo ${ }^{32}$. La National Association of Criminal Défense Lawyers a contribué à cette campagne. Le 10 janvier, dans un communiqué de presse, Amnesty International «salue [welcomes] l'initiative de Benetton, qui pourrait amener une discussion sérieuse au sujet de l'abolition de la peine de mort $»^{33}$. Ce message peut être considéré comme l'expression d'un soutien prudent d'Amnesty International à la campagne de Benetton, mais non d'un réel partenariat entre l'ONG et l'entreprise ${ }^{34}$. Dans un communiqué publié le 28 janvier $^{35}$, Amnesty International (AI) précise d'ailleurs qu'elle n'a pas été impliquée dans la conception de la campagne ni dans la décision de son lancement; AI exprime aussi sa sensibilité pour les victimes de crimes violents et leurs familles et assure qu'elle s'oppose à tout ce qui pourrait inutilement exacerber leur anxiété. Aux Etats-Unis, la campagne suscite de vives 
protestations de parents de victimes et d'organisations en faveur de la peine de mort ${ }^{36}$. Des manifestations ont lieu devant les grands magasins Sears, importants distributeurs de la marque italienne sur le territoire américain. Le 17 février, la direction de Sears annonce qu'elle retire les articles Benetton de ses 400 magasins aux Etats-Unis. En avril 2000, à Genève, des élèves de 12 à 14 ans interpellés par les affiches de la campagne interrogent leur enseignante d'éducation civique sur la peine de mort, ce qui débouche sur un travail en classe et sur un échange suivi de correspondance entre des élèves et des condamnés à mort américains. Le 2 mai à Rome, après dix-huit ans de collaboration, Luciano Benetton remercie Oliviero Toscani, le photographe.

Graphique 1 : Pressions et partenariats entre ONG et entreprises (1974-2000)

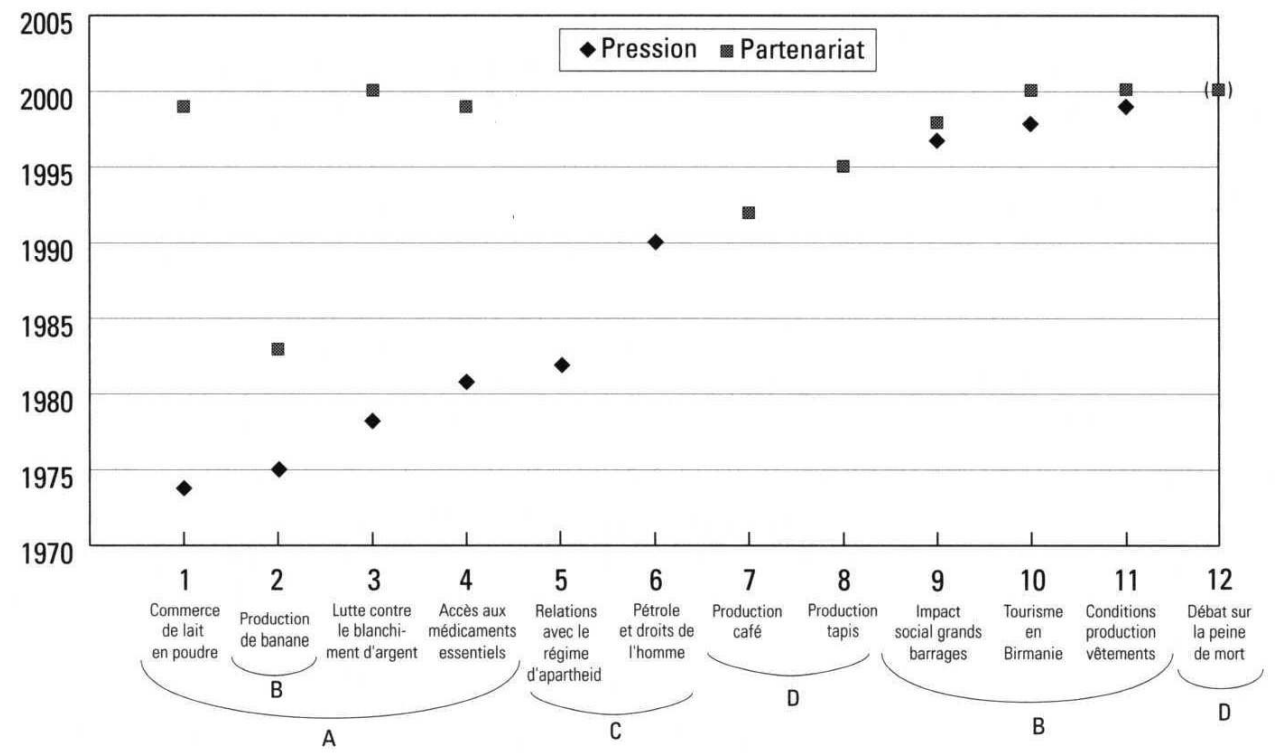

Note : Les carrés et losanges représentant les pressions et les partenariats symbolisent des points de départ et non des événements uniques : l'année indiquée marque le début d'un processus de pression ou de partenariat (cf. date de lancement d'une campagne, de signature d'un accord, etc.).

Le graphique 1 synthétise les 12 cas dans une présentation historique des pressions et partenariats ayant marqué les relations entre ONG et entreprises. Parmi neuf cas de pression, sept ont ensuite fait l'objet d'un partenariat. Cela contredit le préjugé selon lequel ONG et entreprises évoluent forcément dans un rapport antagoniste, et indique que les ONG misent aussi sur la collaboration avec les entreprises pour faire avancer leurs revendications. Cela indique également un rapport historique entre pression et partenariat, qui rejoint une intuition pouvant paraître évidente : la pression précède le partenariat. Une répartition des 12 cas en 4 groupes permet une analyse plus fine.

\section{Groupe A (cas 1, 3, 4) : longue pression avant un partenariat}

Dans ces cas, la relation entre ONG et entreprise demeure longtemps au niveau de la confrontation (1974-1981) avant que ne se négocient des partenariats (1998-2000). Il faut noter que le partenariat ne met pas toujours un terme à la pression, les ONG actives dans les partenariats n'étant pas forcément les mêmes que celles qui animent les campagnes ciblées. Il est courant que les entreprises gèrent d'un côté la pression 
d'ONG « critiques » pendant que d'un autre côté elles développent des partenariats avec des ONG « coopératives ».

\section{Groupe B (cas 2, 9, 10,11) : pressions rapidement suivies d'un partenariat}

Ici, la pression est suivie peu après d'un partenariat (période 1995-2000). Si les campagnes critiques débouchent rapidement sur le dialogue et le partenariat, elles ne s'éteignent pas pour autant : ainsi, la Campagne Clean Clothes (CCC) représente à la fois une pression (visant l'ensemble des marques et distributeurs de vêtements) et un partenariat (trois entreprises participent à un projet pilote d'application du code de conduite (CC). A noter un cas particulier, le cas 2 "Conditions de production de la banane ", dans lequel la pression est rapidement suivie d'un partenariat au début des années 1980 déjà. La Clause sociale Migros-Del Monte est considérée comme un précurseur des partenariats orientés vers l'activité de l'entreprise, tels que les nombreux codes de conduite et labels sociaux apparus dans les années 1990.

\section{Groupe $C$ (cas 5 et 6 ) : pression seulement}

Contrairement à ce qui a été observé pour l'ensemble des autres cas, ONG et entreprises n'ont pas établi de partenariat au sujet des relations avec le régime d'apartheid et du rôle des compagnies pétrolières en matière de droits de l'homme. L'absence de consensus entre les revendications des ONG et la position des entreprises s'observe souvent lors d'appels au boycott liés à la situation des droits de l'homme dans un pays. Dans ces situations, il ne semble pas exister de zone d'intérêt commun entre ONG et entreprises.

\section{Groupe D (cas 7, 8 et 12) : partenariat seulement}

Des partenariats sont noués entre des ONG et des entreprises sans que celles-ci n'aient fait l'objet de pression au préalable, voire sur leur propre initiative. Cela indique l'existence de zones d'intérêt commun entre défenseurs des droits de l'homme et acteurs de l'économie. Le succès du label de commerce équitable Max Havelaar est dans l'intérêt des ONG tout comme dans celui des grands distributeurs qui élargissent ainsi leur gramme de produits et fidélisent de nouveaux consommateurs, sans pour autant rogner sur leur marge. De même, il semble dans l'intérêt d'une ONG comme Amnesty International que la marque Benetton communique sur la peine de mort. Enfin, c'est une association patronale de distributeurs de tapis qui a contacté des ONG pour créer le label STEP. Ces exemples indiquent que des entreprises sont réellement prêtes à prendre des initiatives en matière de droits de l'homme, pour autant que cette démarche s'inscrive dans leur vocation d'entités privées soumises à concurrence et à la contrainte de la rentabilité.

\section{Pression des ONG et bilan social de l'entreprise}

Le graphique 1 montre que dans sept cas sur neuf, la pression est suivie d'un partenariat. Ce résultat exprime-t-il pour autant un réel pouvoir des ONG sur les 
entreprises? La pression ciblée des ONG exerce-t-elle un effet de levier sur le comportement des entreprises? Un bilan social des 28 entreprises du Swiss Market Index a été dressé en mai $2001^{37}$ : on a cherché à estimer la quantité de communications fournies par l'entreprise pour décrire l'impact économique, social et environnemental de ses activités, en particulier sur les pays émergents et en développement. Des différences sensibles ont été observées parmi les résultats obtenus: certaines entreprises présentent un grand nombre d'informations sous une rubrique Développement durable ou Notre responsabilité envers la société, dans lesquelles elles expliquent comment elles traitent leurs collaborateurs, ménagent la biodiversité, s'engagent auprès de leurs fournisseurs pour améliorer les conditions de travail, soutiennent des projets de développement, etc. D'autres entreprises se contentent de mentionner quelques donations caritatives et opérations de sponsoring humanitaire sans dévoiler grand chose de leurs activités. Comment expliquer ces différences de bilan social parmi les 30 plus grandes entreprises suisses? Pour répondre à cette question, on a cherché à établir une corrélation entre le bilan social et différentes variables. Quatre variables ont été testées : pression sociale, part des ventes dans les pays en développement, capitalisation boursière, performance boursière.

Graphiques 2-5: Comment expliquer les différences de bilan social ? Test sur le Swiss Market Index (juin 2001) 


\section{Graphique 2 : Bilan social en fonction de la pression sociale}

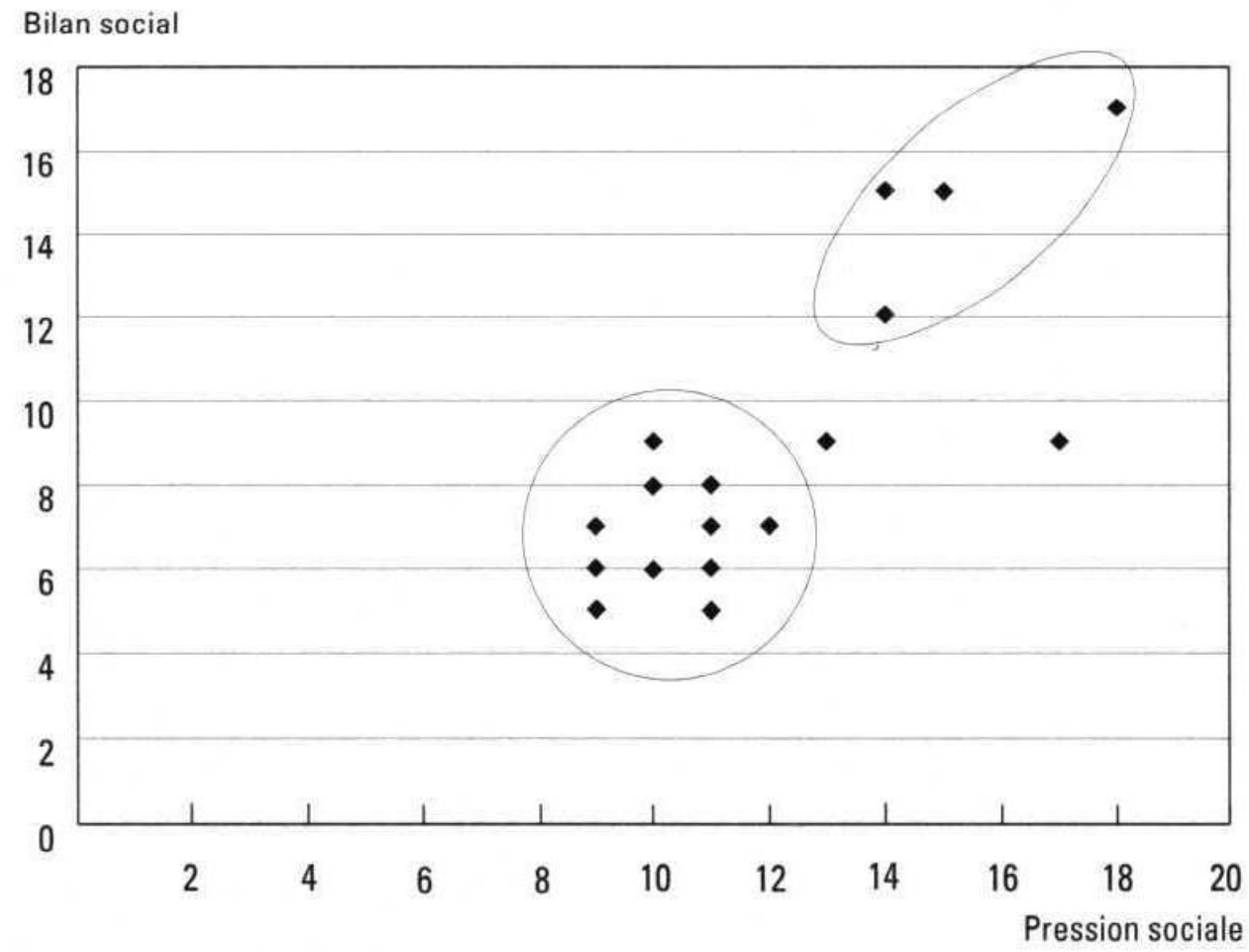

BILAN SOCIAL : estimation de la quantité de communications fournies par l'entreprise pour décrire son impact social.

PRESSION SOCıLE : estimation de la quantité de communications fournies par la société civile (ONG, universités, agences internationales, médias...) pour décrire l'impact social de l'entreprise. On observe une relation positive entre bilan social et pression sociale, ce qui indique que les entreprises les plus critiquées et sollicitées par les ONG sont celles qui présentent le bilan social le plus consistant. Ce résultat vient confirmer et élargir le précédent : non seulement la pression des ONG amène les entreprises à s'engager dans des partenariats, elle les incite aussi à agir de manière individuelle afın d'améliorer la perception et l'évaluation de leur performance sociale. Cela montre que lorsqu'elles exercent une pression ciblée, les ONG disposent d'un pouvoir réel sur les entreprises. On constate que 4 entreprises (en haut à droite) sont l'objet de multiples pressions et affichent un bon bilan social, alors que la majorité des entreprises se situe dans une position inverse peu de pression, faible bilan social (en bas au milieu) 
Graphique 3 : Bilan social en fonction des ventes dans les PVD

Bilan social

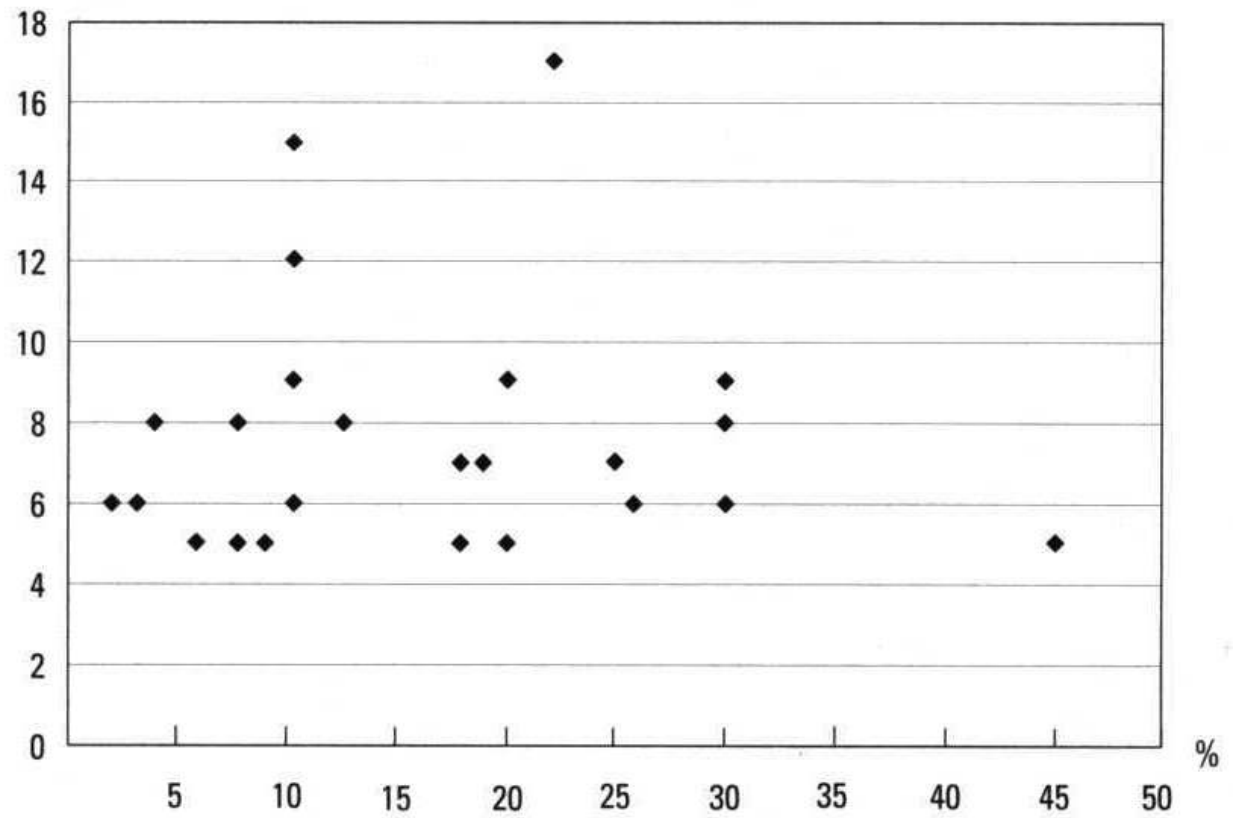

Pas de relation apparente entre bilan social et part du chiffre d'affaires réalisé dans les pays en développement (PVD) : des entreprises qui vendent essentiellement au Nord prennent plus d'initiatives sociales que des entreprises qui réalisent près de la moitié de leur chiffre d'affaires dans le Sud.

Graphique 4 : Bilan social en fonction de la capitalisation boursière

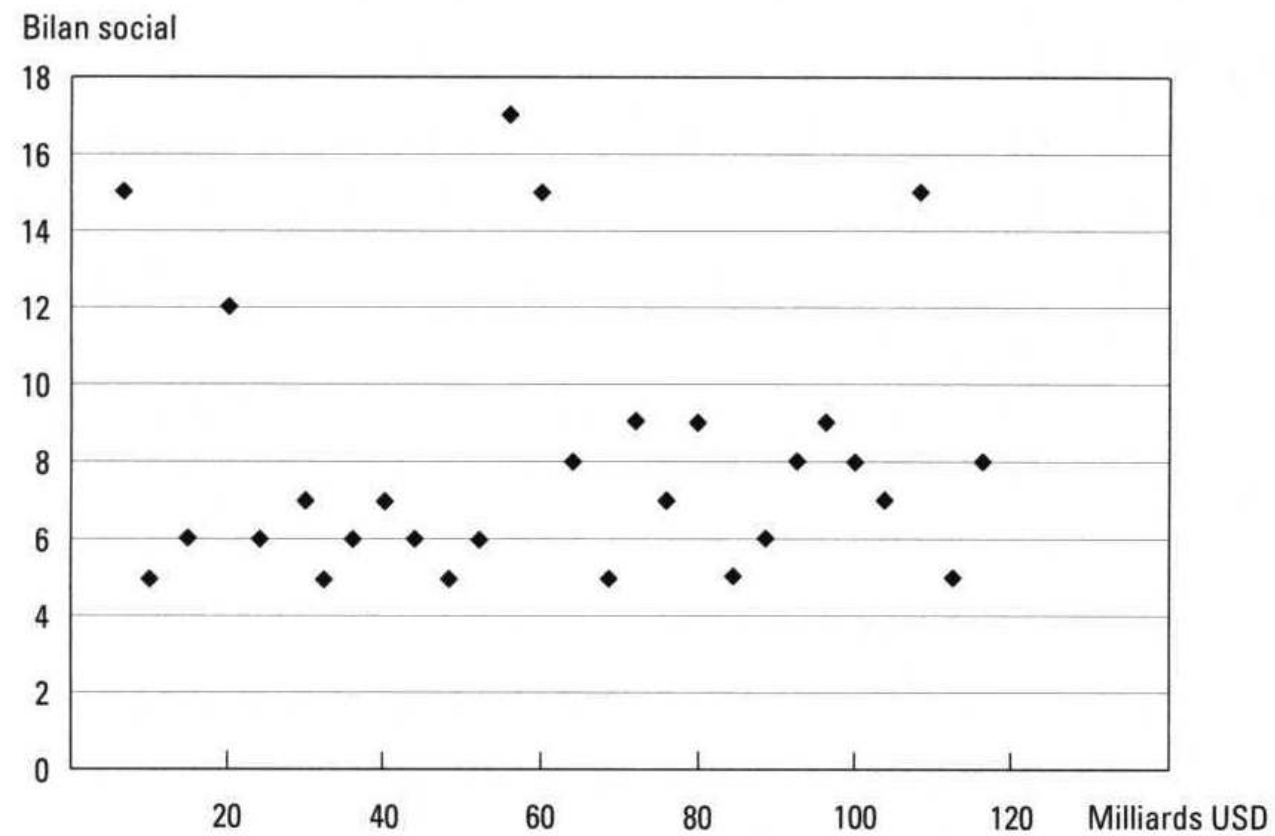

Pas de relation apparente entre bilan social et taille de l'entreprise, indiquée par sa capitalisation boursière : on constate que des entreprises de taille moyenne prennent plus d'initiatives sociales que des grandes. 
Graphique 5 : Bilan social en fonction de la performance boursière

Bilan social

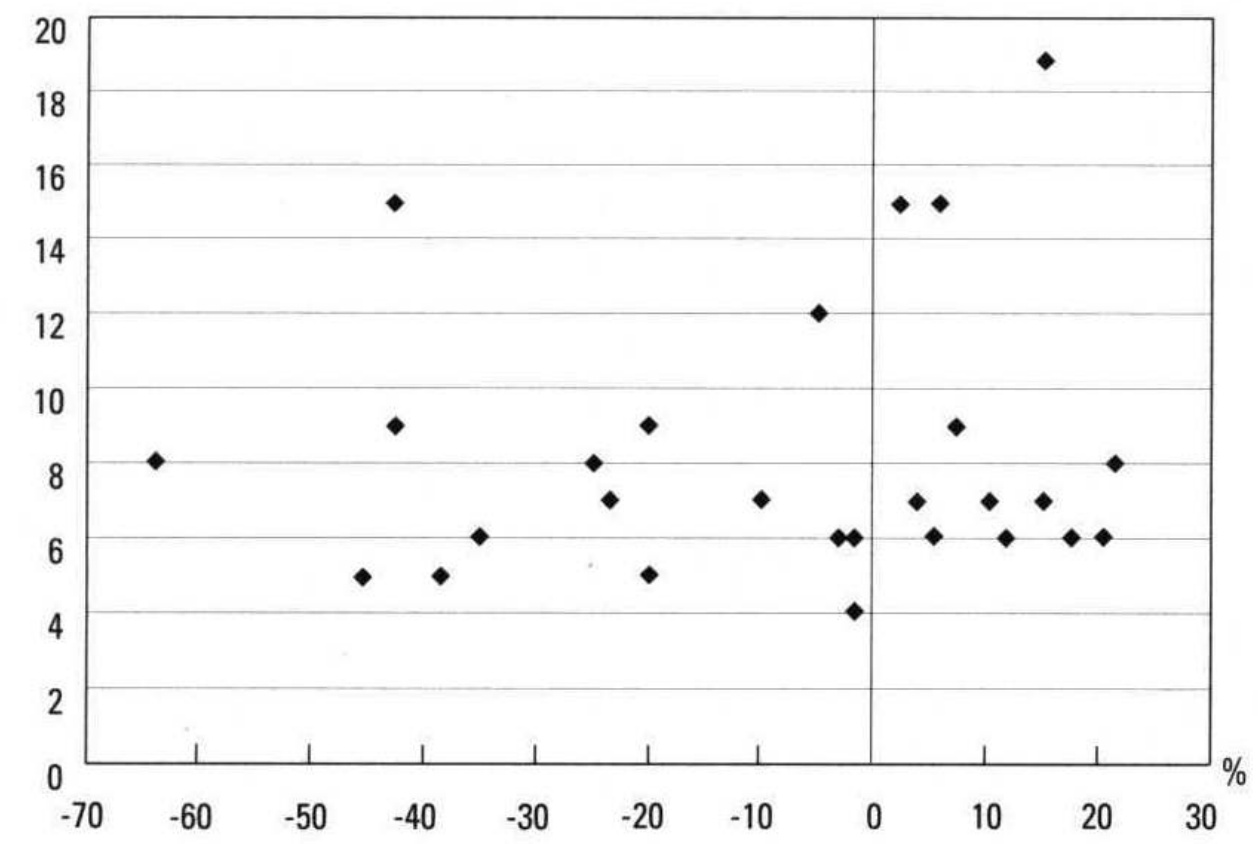

Pas de relation apparente entre bilan social et performance boursière : on ne peut pas dire que le rendement financier amène la performance sociale, ni que la performance sociale amène le rendement financier.

Note : ces graphiques n'indiquent pas le nom des entreprises, parce qu'ils reposent sur des données incomplètes et représentent des résultats d'un caractère trop provisoire.

\section{Impact des ONG sur l'évaluation de la performance sociale de l'entreprise}

L'investissement socialement responsable ", ou "investissement éthique ", apparait aux Etats-Unis dans les années 1920, lorsque des communautés religieuses décident de placer leur argent en conformité avec leurs convictions, ce qui débouche sur des placements excluant les entreprises impliquées dans la vente d'alcool, la pornographie ou les jeux d'argent. Variante plus récente de l'investissement éthique de type exclusif, les "fonds verts", apparus en Europe à la fin des années 1980, prohibent les investissements dans l'industrie automobile, agroalimentaire, l'énergie nucléaire, etc. Une approche inclusive de l'investissement éthique s'est développée parallèlement, également sous l'impulsion des Eglises américaines: l'« activisme actionnarial», ou " actionnariat socialement responsable ». Cette approche amène des ONG à acquérir quelques actions pour faire entendre leur voix dans les assemblées générales et auprès de la direction d'entreprises jugées socialement perfectibles (cf. en Suisse CANES avec Nestlé, l'Association des actionnaires critiques de l'UBS, la Déclaration de Berne avec ABB, Actares).

Le modèle d'investissement éthique exclusif présente deux inconvénients : d'un point de vue financier, il oblige à réduire la répartition des risques et les opportunités de rendement; d'un point de vue éthique, il empêche de promouvoir des innovations sociales progressives auprès d'industries qui sont jugées définitivement fautives. Sous 
l'influence du concept de développement durable, une nouvelle catégorie de produits d'investissement socialement responsable apparaît dans les années 1990, le modèle du "premier de classe " (best-of-class) : on n'exclut aucune industrie a priori mais on s'efforce de valoriser, dans chaque secteur, les entreprises présentant le meilleur profil social et environnemental ; cela rejoint, dans un esprit de réformisme pragmatique, la démarche d'un exercice actif des droits de vote d'actionnaires. Les produits s'inscrivant dans cette tendance nécessitent des évaluations systématiques et nuancées de la performance sociale de l'entreprise, que des instituts spécialisés fournissent aux banques et investisseurs. Plusieurs indices de durabilité reposant sur une telle approche ont été lancés ces dernières années, comme Dow Jones Sustainability Index et FTSE4Good.

L'« investissement durable», forme contemporaine de l'investissement éthique, commence à intéresser les acteurs financiers conventionnels, qui voient dans l'évaluation de la performance sociale de l'entreprise une mesure de sa réputation et de précieuses indications sur les risques qui la caractérisent. Cette évolution ne va pas sans dérouter bon nombre d'observateurs, tant du côté des promoteurs de l'éthique que de celui des investisseurs professionnels, les uns et les autres étant plutôt habitués à percevoir leurs intérêts comme opposés. Des banques telles que Sarasin, UBS ou Lombard Odier se sont dotées d'équipes d'analystes en développement durable qui passent au crible les titres intéressant leurs clients investisseurs, mais aussi les opérations de la banque elle-même, sous l'angle de la durabilité. Ainsi, que leur motivation première soit d'ordre éthique ou financier, de plus en plus d'investisseurs font appel à des analyses sociales et environnementales des entreprises pour compléter les analyses purement économiques.

Certaines banques procèdent elles-mêmes à des évaluations de performance sociale, d'autres font appel à des consultants extérieurs. Comment mesure-t-on le degré d'éthique d'une multinationale? On s'adresse d'abord aux entreprises elles-mêmes, qui publient des rapports sociaux et environnementaux et répondent à de nombreux et longs questionnaires. Mais comment s'assurer que les réponses obtenues reflètent la réalité, que l'éthique affirmée est l'éthique pratiquée ? Et parmi les différentes éthiques défendues dans le monde, laquelle prendre comme référence? Les boycotts de pays pour cause de violation des droits de l'homme servent-ils la cause qu'ils défendent? Condamnés par les ONG et les consommateurs du Nord, les organismes génétiquement modifiés (OGM) ne représentent-ils pas une chance pour l'agriculture et le développement du Sud ? Et les grands barrages? Les agences de notation n'ont pas la prétention de détenir les réponses aux interrogations qui animent les experts du développement économique et social. Elles se tournent donc vers les émetteurs de normes éthiques, vers les gouvernements et les ONG, vers les agences de l'ONU et les universités, et se débrouillent avec les différents messages enregistrés, reflets variés du pluralisme caractéristique de la société moderne (ou postmoderne).

Les ONG participent, au moins indirectement, à l'évaluation de la performance sociale de l'entreprise, jouant à cet égard un rôle d'expert involontaire. Des enjeux comme l'impact social des grands barrages, la lutte contre le blanchiment d'argent, le commerce de lait pour bébé, les OGM ou les relations avec la Birmanie sont pris en compte par les instituts d'évaluation sociale au moment d'estimer les entreprises concernées. De même, lorsqu'une ONG et une entreprise établissent un partenariat, celui-ci représente une information pertinente dans le cadre de l'évaluation de la 
performance sociale de l'entreprise, et pèse sur la note attribuée par les instituts spécialisés.

Des ONG participent aussi directement à l'évaluation de la performance sociale de l'entreprise, jouant alors volontairement un rôle d'expert. Eiris, base de données sur la responsabilité sociale de l'entreprise gérée à Londres et partenaire des indices FTSE4Good, a été fondée par un réseau d'ONG. En Suisse, Centre Info (Fribourg), bureau de consultant en investissement durable, a lui aussi été fondé par des personnes actives dans les milieux du développement, tout comme, plus récemment, Covalence (Genève). Enfin, dernier exemple, une série de banques suisses et européennes développent des Indicateurs de performance sociale pour l'industrie financière, consultant pour cela différentes parties prenantes, parmi lesquelles des $\mathrm{ONG}^{38}$.

\section{Evaluation du pouvoir des ONG sur les entreprises selon le type de relation}

L'analyse du rôle indirect et direct des ONG dans le processus d'évaluation de la performance sociale de l'entreprise nous permet de compléter la typologie des relations entre ONG et entreprises esquissée en début d'article (tableau 1).

Tableau 1 : Typologie des relations entre ONG et entreprises : pression, partenariat, évaluation

\begin{tabular}{|c|c|c|c|}
\hline \multicolumn{2}{|c|}{$\begin{array}{l}\text { Types de relations entre } \\
\text { ONG et entreprise }\end{array}$} & Illustrations & Rôle des ONG \\
\hline \multirow{3}{*}{ Pression } & Institutionnelle & $\begin{array}{l}\text { Lobbying dans les institutions } \\
\text { nationales et internationales }\end{array}$ & \multirow{3}{*}{ Critique } \\
\hline & Informelle & Manifestations, de Seattle à Gênes & \\
\hline & Ciblée & Campagnes de sensibilisation, boycotts & \\
\hline \multirow[t]{2}{*}{ Partenariat } & $\begin{array}{l}\text { Orienté vers } \\
\text { l'activité de } \\
\text { l'entreprise }\end{array}$ & $\begin{array}{l}\text { Labels, codes de conduite, } \\
\text { certification sociale, soutien à } \\
\text { une initiative individuelle }\end{array}$ & Soutien/expert \\
\hline & $\begin{array}{c}\text { Orienté vers } \\
\text { l'activité de l'ONG }\end{array}$ & Sponsoring, marketing lié à une cause & Soutien \\
\hline \multirow{2}{*}{ Evaluation } & Indirecte & $\begin{array}{l}\text { Les campagnes de pression et les } \\
\text { partenariats reliant ONG et entreprises } \\
\text { sont pris en compte par les organes } \\
\text { d'évaluation de la performance } \\
\text { sociale de l'entreprise }\end{array}$ & \multirow[t]{2}{*}{ Expert } \\
\hline & Directe & $\begin{array}{l}\text { Des ONG créent, ou collaborent avec, } \\
\text { des agences d'évaluation de la } \\
\text { performance sociale des entreprises }\end{array}$ & \\
\hline
\end{tabular}

\section{Pression institutionnelle}

Dans le cadre des projets menés auprès des trois institutions mentionnées - clause sociale à l'OMC, Principes directeurs de l'OCDE à l'attention des entreprises multinationales et Groupe de travail de la Sous-commission des droits de l'homme des 
Nations Unies sur les entreprises transnationales -, on tente de promouvoir l'adoption de normes légalement contraignantes à l'égard des entreprises en matière de droits de l'homme. Les opportunités politiques paraissent ici limitées, comme pour ce qui concerne la contrainte vis-à-vis des Etats dans le même domaine. Selon le Rapport mondial sur le développement humain publié par le PNUD en 2000, «il n'y a pas grandchose dans l'ordre mondial actuel qui contraigne les Etats et les acteurs mondiaux à défendre les droits de l'homme à l'échelle planétaire ${ }^{39}$. Cela illustre le vaste problème de l'adoption et de la mise en œuvre de politiques publiques de portée globale dans un monde où prévalent les souverainetés nationales. Précisons que le thème de la pression institutionnelle n'a été qu'effleuré dans cette recherche.

La relation des entreprises avec la problématique des droits de l'homme recouvre deux réalités aux implications juridiques et politiques différentes: respect, promotion. Comme tous les organes de la société (Préambule à la Déclaration universelle des droits de l'homme), les entreprises sont tenues de respecter - de ne pas violer - les droits de l'homme. Si une entreprise recourt au travail forcé dans une de ses filiales, si elle commandite la répression violente d'une manifestation syndicale ou si elle mène des expérimentations dangereuses sur des cobayes humains non consentants, elle devra normalement rendre des comptes à la justice. Des normes légales existent. D'autre part, les entreprises sont aussi appelées à promouvoir les droits de l'homme, c'est-à-dire à agir auprès de tiers (gouvernements, entreprises et autres parties prenantes) pour les inciter à eux-mêmes respecter les droits de l'homme; au-delà, les entreprises sont appelées à participer à la réalisation des droits de l'homme, dans des situations qui dépassent l'alternative respecter/violer, et qui concernent notamment les droits économiques, sociaux et culturels : les droits à l'alimentation, à la santé, au logement ou à l'eau potable ne sont pas seulement des droits que l'on ne doit pas violer, ils sont aussi, et peut-être surtout, des droits que l'on doit réaliser, concrétiser, promouvoir, ce qui oblige à concevoir un large champ d'action, une multiplicité d'acteurs et, peut-être, des réglementations de mise en œuvre spécifiques, sans remettre en cause le principe de l'indivisibilité des droits de l'homme.

Dans la plupart des 12 cas passés en revue, l'entreprise n'est pas appelée à respecter elle-même les droits de l'homme, mais à promouvoir le respect et la réalisation des droits de l'homme auprès de différentes parties prenantes. Cela indique que même si l'entreprise n'est pas considérée comme un coupable potentiel aux yeux de la loi, on considère cependant que sa présence globale l'oblige moralement à intervenir dans des questions sociales et politiques lointaines. Pour l'Etat, et pour les acteurs qui s'efforcent de peser sur les décisions gouvernementales, il y a là matière à adopter d'intéressantes mesures d'incitation à la responsabilité sociale de l'entreprise. Quelques exemples : la Garantie suisse contre les risques à l'exportation soumet aux candidats un questionnaire socio-environnemental; la Confédération (Seco) a soutenu dès sa création la Fondation Max Havelaar; des municipalités américaines ont adopté des politiques d'achat publics tenant compte du comportement des entreprises en matière de droits de l'homme et de droit du travail; les caisses de retraite britanniques, australiennes et (prochainement) allemandes sont soumises à l'obligation d'informer le public de leur politique d'investissement et d'indiquer si elles appliquent ou non des critères de durabilité à leurs placements. L'intensification de ce type de mesures dépendra de nombreux facteurs, dont, certainement, l'évolution de la culture politique des partis dominants et de celle des ONG, syndicats et mouvements sociaux. Il est à 
prévoir que gouvernements et ONG travailleront de plus en plus de concert dans le but de stimuler la responsabilité sociale de l'entreprise.

\section{Pression informelle}

Ce type de relation ne semble pas entraîner d'effets immédiats sur le comportement des entreprises. En revanche, il est possible qu'il ait une influence sur d'autres modes de relations entre ONG et entreprises: pression institutionnelle, pression ciblée, partenariats. Dans cette hypothèse, les manifestations antimondialisation et les échos médiatiques qu'elles suscitent créent un climat qui stimule les initiatives relevant de la responsabilité sociale de l'entreprise et, peut-être, l'adoption de réglementations dans ce domaine. A l'inverse, en véhiculant une critique systématique des multinationales et en leur prêtant un visage diabolique, ces manifestations risquent d'inhiber des énergies citoyennes, de voiler l'espace politique qui existe entre rébellion et résignation (voir notre conclusion).

\section{Pression ciblée}

Les campagnes de pression exercées par les ONG sont presque toujours suivies de partenariats associant l'entreprise visée et des ONG (celles qui ont animé la campagne ou d'autres). Cela représente de réelles opportunités politiques, sous réserve bien sûr de l'utilité et de l'ampleur des initiatives prises dans le cadre du partenariat, ce qui pose la question du contrôle (interne, externe, indépendant?) de l'application de mesures volontaires et celle de leur évaluation. Plus largement, on a observé que les entreprises les plus critiquées et sollicitées par les ONG sont celles qui présentent le bilan social le plus complet. Cela illustre une des limites des campagnes de pression: celles qui se focalisent année après année sur une seule entreprise, généralement un leader de la branche, risquent de favoriser une responsabilité sociale à deux vitesses entre les quelques bons élèves qui ont tiré la leçon des coups de bâton accumulés et le reste de la classe qui, épargné par les foudres des ONG, prend du retard dans une relative indifférence. De ce point de vue, les campagnes qui tendent à élargir leur cible sont à considérer avec un intérêt particulier.

\section{Partenariat orienté vers l'activité de l'entreprise}

Les entreprises semblent de plus en plus désireuses de nouer des partenariats avec des ONG, opérations qui autorisent de précieux transferts d'image. Pour les ONG, il y a là un moyen de participer de l'intérieur à un ajustement des pratiques d'une entreprise en amenant leur expertise, ce qui, dans le principe, représente des opportunités politiques intéressantes. La question de l'engagement dans un partenariat avec une entreprise, et du risque possible de perte d'indépendance qui y est souvent associé, est considérée différemment par les ONG selon qu'elles sont "politiques » (sensibilisation du public, lobbying) ou «de terrain» (financement de projets). Pour les organisations qui sont actives sur les deux fronts, la question de l'opportunité des partenariats avec le secteur privé est particulièrement délicate. Cela dit, de nombreux partenariats entre des ONG et des entreprises ont permis à celles-ci d'améliorer leur impact social ou environnemental. L'avis d'un représentant d'Helvetas: «Nous sommes ouverts aux 
partenariats avec les entreprises. Il faut travailler avec les multinationales, les aider à améliorer leur contribution au développement. $»^{40}$

\section{Partenariat orienté vers l'activité de l'ONG}

Depuis quelques années, les opérations de sponsoring et de marketing lié à une cause associant entreprises et ONG se multiplient, principalement du côté des «ONG de terrain », parfois de celui des «ONG politiques ». Les opportunités politiques résident ici dans l'ampleur et l'utilité des ressources attribuées par l'entreprise à des projets sociaux à travers le sponsoring ou le marketing lié à une cause. On a vu par exemple des entreprises pharmaceutiques consentir à d'importantes donations de médicaments, ou des géants de l'informatique s'impliquer substantiellement dans des programmes visant la réduction de la pauvreté. En fait, à peu près toutes les entreprises disposent de programmes de donations caritatives, plus ou moins étendus, plus ou moins objets de communication.

\section{Evaluation indirecte}

Lorsque les ONG lancent une campagne critique visant une entreprise, ou lorsqu'elles soutiennent une initiative de l'entreprise dans le cadre d'un partenariat, cela est pris en compte dans le secteur financier. Les ONG pèsent en effet sur l'évaluation de la performance sociale de l'entreprise, et donc sur la pondération des titres composant les produits d'investissement intégrant des critères de durabilité, ainsi que sur l'analyse et la gestion des risques de réputation. L'action de l'ONG sur l'entreprise est double : la campagne/partenariat agit directement sur l'entreprise à court terme, mais aussi de façon indirecte, à plus long terme, via la sensibilisation du public, les relais médiatiques, la perception des consommateurs et certaines stratégies d'investissement. Ces phénomènes, et les opportunités politiques correspondantes, devraient être considérés avec soin par les ONG, qui disposent d'une sympathie et d'une écoute grandissantes dans le public et les médias, ainsi que par les autres acteurs du développement.

\section{Evaluation directe}

Certaines ONG participent directement et plus largement à l'évaluation de la performance sociale de l'entreprise en mettant sur pied des bases de données et des agences de notation au service des investisseurs. Promoteurs du développement, des droits de l'homme, de la paix ou de la protection de l'environnement, les ONG (et les gouvernements) peuvent enrichir l'évaluation de la performance sociale de l'entreprise, y intégrer leurs préoccupations, lui donner de la vigueur. Ancrées dans la société civile, elles peuvent convaincre les citoyens (et les médias, partis, syndicats, caisses de pension) d'actionner les leviers que sont le consumérisme éthique et l'investissement durable. Pour cela, certaines ONG appuient, en leur prêtant une part de leur image, des mécanismes d'évaluation/certification/label. Ce domaine recèle des opportunités politiques complémentaires aux processus institutionnels qui méritent certainement d'être étudiées. 


\section{Conclusion : des opportunités politiques à saisir}

41 Les campagnes de pression directe incitent les entreprises à établir des partenariats avec des ONG afin d'améliorer leur performance sociale, ainsi qu'il ressort de l'étude d'une douzaine d'enjeux socio-économiques de dimension globale. L'analyse du bilan social des 28 titres du Swiss Market Index indique plus généralement que les entreprises les plus critiquées et sollicitées par les ONG sont celles qui présentent le bilan social le plus étendu. Cela témoigne du pouvoir exercé par les ONG sur les multinationales dans le passé et cela trace des opportunités politiques futures. Les ONG entretiennent trois types de relations avec les entreprises, auxquels correspondent autant de rôles: pression (rôle critique), partenariat (rôle de soutien), évaluation (rôle d'expert). Alors que le secteur financier tient de plus en plus compte des actions des ONG dans ses analyses, les ONG découvrent un nouveau champ d'action politique : le consumérisme éthique, l'évaluation de la performance sociale de l'entreprise, l'investissement durable. Pour amener les entreprises à jouer un plus grand rôle en matière de droits de l'homme et de développement, les stratégies fondées sur la contrainte légale sont insuffisantes. Les opportunités politiques résident aussi dans une relation directe avec les entreprises, une pression différenciée qui peut impliquer la négociation, le partenariat, l'évaluation, et le recours à des réalités économiques telles que la compétition, la recherche du profit, la valeur des marques, les relations publiques (cf. Luhmann 1989).

La critique systématique des multinationales est contre-productive par rapport aux valeurs qui la motivent. A son insu, elle risque en effet d'inhiber le potentiel d'engagement des citoyens employés dans, clients et actionnaires de ces entreprises, ou plutôt elle court le risque de s'en éloigner, de les éloigner et de s'éloigner elle-même de la chose publique. Un constat réaliste est plus motivant : les entreprises contribuent au développement humain, mais elles peuvent aussi l'entraver, et surtout elles pourraient y contribuer davantage. Elles sont, comme les hommes qui les constituent, imparfaites et capables de changer. Il faut donc différencier la perception de l'activité économique, s'informer, évaluer, stimuler. Cela demande au préalable de s'approprier psychologiquement les multinationales, d'en normaliser les représentations, de les observer avec curiosité et singularité, de les approcher, de les assumer.

Les citoyens d'aujourd'hui sont décrits comme peu politisés, individualistes, repliés sur leur intimité. Qu'ils trouvent les multinationales sympathiques ou diaboliques, l'attitude est passive : elles font partie du paysage. Ils n'en ignorent pas pour autant les valeurs de justice sociale, la solidarité, la fraternité, comme s'ils les avaient reniées. Ces valeurs s'expriment à l'occasion de votations et de manifestations, ainsi que dans le comportement quotidien, la famille, la rencontre informelle. On peut trouver que cela n'est pas assez, et peut-être qu'en effet des citoyens se sentent frustrés, qu'ils aimeraient en faire plus, ou autrement, et que se méfiant des carcans militants ils ressentent un manque d'engagement, parce qu'ils s'imaginent que la politique commence là où finit leur liberté, parce qu'ils n'ont pas beaucoup de temps. Ne faudrait-il pas, à ces citoyens, donner l'occasion d'agir par des moyens politiquement efficients et adaptés à leur mode de vie, les rapprocher des leviers de changement social sans inquiéter leur confort, mettre à leur disposition un programme politique user-friendly? Encore balbutiants, le consumérisme éthique, l'évaluation de la performance sociale de l'entreprise et l'investissement durable font partie de ces 
moyens. Il vaut la peine que les gouvernements, universités et ONG y consacrent de l'attention et des ressources dans le but de les intensifier et de les orienter, quitte à prêter une part de leur légitimité aux mécanismes les plus pertinents, car il y a là matière à élargir les possibilités d'action démocratique.

\section{BIBLIOGRAPHIE}

Avery Christopher L., 2000, Business and Human Rights in a Time of Change, Amnesty International UK.

Bendell Jem, 2000, Terms for Endearment : Business, NGOs and Sustainable Development, Greenleaf Publishing Limited in association with The New Academy of Business.

Elkington John and Shelly Fennell, 1998, « Partners for Sustainability », Greener Management International, Issue 24.

Joerchel Bastienne, 1997, «Les organisations de protection de l'environnement et le secteur économique : de la confrontation à la coopération », in Benninghoff Martin, Bastienne Joerchel, Peter Knoepfel (dir.), L'écobusiness : enjeux et perspectives pour la politique de l'environnement, Basel and Frankfurt am Main : Helbing \& Lichtenhahn.

Luhmann Niklas, 1989, Ecological Communication, Cambridge University Press.

Mach Antoine, 2001, Entreprises suisses et droits de l'homme : confrontations et partenariats avec les ONG, Editions universitaires de Fribourg - Antenna Internationale, Institut interdisciplinaire d'éthique et des droits de l'homme.

Teubner Gunther, 1997, Global Law without a State, Aldershot : Dartmouth Gower.

Utting Peter, 2001, «La responsabilité des entreprises dans la perspective d'un développement durable », Annuaire Suisse-Tiers Monde 2001, Genève : Institut universitaire d'études du développement, pp. 110-132.

\section{NOTES}

1. Cet article reprend les résultats d'une recherche menée sur mandat de la Fondation Antenna internationale (Mach 2001), ainsi que des travaux réalisés ultérieurement au sein de Covalence SA, Genève.

2. Cf. CETIM (Centre Europe - Tiers Monde), Les activités des sociétés transnationales et la nécessité de leur encadrement juridique, Actes et conclusions du séminaire de Céligny, 4 et 5 mai 2001, www.cetim.ch/activ/activfra.htm.

3. www.oecd.org/daf/investment/guidelines/indexf.htm.

4. Cf. Joerchel Anhorn Bastienne, «Les principes directeurs pour multinationales de l'Organisation de coopération et développement économiques (OCDE)», Annuaire Suisse - Tiers Monde 2001, Genève : Institut universitaire d'études du développement, pp. 101-109.

5. War on Want, Arbeitsgruppe Dritte Welt Bern, Infact, International Baby Food Action Network, La Déclaration de Berne, CANES (Convention d'actionnaires Nestlé), etc. 
6. International Baby Food Network, Breaking the Rules, 1994, cité dans Ethical Consumer Critic Database, www.ethicalconsumer.org, 2000.

7. Nestlé, Rapport sur les actions entreprises en relation avec le Code international del'OMS, $\mathrm{n}^{\circ} 4$, avril 2000, www.babymilk.nestle.com.

8. Titre d'un livre écrit par Ursula Brunner, Frauenfeld/Stuttgart/Wien: Verlag Huber, 1999.

9. Pain pour le prochain, Entraide protestante, avec le soutien de la Déclaration de Berne, Helvetas, Swissaid et Action de Carême.

10. Cf. Stuckelberger Christoph and Michel Egger, «A Social Clause with a TNC: The Migros-Del Monte Case ", Swiss Coalition News 7, Communauté de travail des œuvres d'entraide, mars 1996, p. 6.

11. Ce manifeste a été soutenu, notamment, par Action de Carême, Helvetas, Entraide protestante, Pain pour le prochain, Swissaid, Terre des Hommes - Suisse.

12. www.drugdonations.org.

13. www.foundation.novartis.com.

14. www.gIobalforumhealth.org.

15. Mouvement anti-apartheid de Suisse, Déclaration de Berne, Action de Carême, Pain pour le prochain, CANES, Action Place financière Suisse, Association des actionnaires critiques de l'UBS, etc.

16. Groupe interdépartemental Suisse - Afrique du Sud, Les relations entre la Suisse et l'Afrique du Sud, Berne : Confédération suisse, juillet 1999, www.dfe.admin.ch/dynamic/Presse_Rohstoff/ CH_RSA/F/af_sud_ 1 f.htm.

17. Groupe de travail Afrique australe - Terre nouvelle - des œuvres protestantes d'entraide et de mission et la Table ronde Afrique australe du Conseil missionnaire catholique suisse, Suisse Afrique du Sud. Faire mémoire pour assurer le présent, Matran, 14-15 janvier 2000, mai 2000.

18. Sané Pierre, Al and Economie Actors, May 30, 2001, www.amnesty.org.

19. www.shellnigeria.com/shell/hr_rhs.asp.

20. www.maxhavelaar.ch ; Max Havelaar Foundation, Swiss Coalition News 5, septembre 1995.

21. www.maxhavelaarfrance.org/label4.html, chiffres 2000.

22. www.step-foundation.ch.

23. www.ladb.ch.

24. Pour la position du Secrétariat à l'économie au sujet de la Garantie contre les risques à l'exportation liée à la centrale des Trois Gorges en Chine, www.admin.ch/cp/f/ 37E7A6DE.CB056EF4@seco.admin.ch.html.

25. www.dams.org.

26. www.birmanie.int.ch/ asb.

27. www.akte.ch.

28. Michel Danthe, directeur des publications de la presse Migros, réponse aux membres de AKTE, 23 juillet 1998, cité in Mach 2001.

29. Entretien avec Danièle Gosteli, groupe Economie et droits humains, Amnesty International Suisse, 16 novembre 2001.

30. www.tourismfordevelopment.com.

31. www.cleanclothes.ch.

32. Benetton, Looking at Death in the Face, Communiqué de presse, Ponzano, 7 janvier 2000, www.benetton.com/wws/aboutyou/campinfo/index.html.

33. Amnesty International's Position regarding Benetton's Worldwide Campaign about the Death Penalty, 10 January 2000, www.web.amnesty.org/ai.nsf/index/ACT500022000.

34. Raison pour laquelle le symbole figurant sur le graphique 1 est mis entre parenthèses.

35. Amnesty International's Reaction to Benetton's Ongoing Campaign against the Death Penalty, Press release, 28 January 2001, www.web.amnesty.org/ai.nsf/index/ACT500032000. 
36. Cf. National Organization of Parents of Murdered Children, Press release, 11 January 2000, www.pomc.com/minealertl,cfm; www.prodeathpenalty.com.

37. Adecco, ABB, Bâloise, Ciba SC, Clariant, CSG, EMS, Givaudan, Holcim, Julius Baer, Kudelski, Lonza, Nestlé, Novartis, Rentenanstalt, Richemont, Roche, Serono, SGS, Sulzer, Syngenta, Swatch, Swisscom, Swiss Re, Swissair, UBS, Unaxis, Zurich.

38. Social Performance Indicators for the Financial Industry, www.spifmance.com.

39. Rapport mondial sur le développement humain 2000, Programme des Nations Unies pour le développement, de Boeck Université, 2000.

40. Entretien avec Tobias Meier, directeur des ventes, Helvetas, 9 octobre 2001.

\section{AUTEUR}

\section{ANTOINE MACH}

Politologue, responsable de la recherche, Covalence SA, Genève. 